\title{
Construction de l'argumentation dans l'article de recherche en mécanique, différences entre discours du francophone et de l'anglophone
}

Patrick Bachschmidt

\section{(2) OpenEdition}

Journals

Édition électronique

URL : http://journals.openedition.org/asp/2418

DOI : $10.4000 /$ asp. 2418

ISBN : 978-2-8218-0380-0

ISSN : 2108-6354

\section{Éditeur}

Groupe d'étude et de recherche en anglais de spécialité

\section{Édition imprimée}

Date de publication : 1 décembre 1999

Pagination : 197-208

ISSN : 1246-8185

Référence électronique

Patrick Bachschmidt, «Construction de l'argumentation dans l'article de recherche en mécanique différences entre discours du francophone et de l'anglophone », ASp [En ligne], 23-26 | 1999, mis en ligne le 27 juin 2011, consulté le 19 avril 2019. URL : http://journals.openedition.org/asp/2418 ; DOI : 10.4000/asp.2418

Ce document a été généré automatiquement le 19 avril 2019

Tous droits réservés 


\title{
Construction de l'argumentation dans l'article de recherche en mécanique, différences entre discours du francophone et de l'anglophone
}

\author{
Patrick Bachschmidt
}

\section{Introduction}

1 Nombreuses sont les publications scientifiques rédigées en anglais par des chercheurs francophones qui sont rejetées par les comités de lecture, non pas tant en raison de fautes d'orthographe ou de grammaire, que " parce que cela ne fait pas anglais ».

2 J'enseigne la communication en anglais à des élèves-ingénieurs à l'Université de HauteAlsace et suis ainsi constamment sollicité par mes collègues scientifiques, enseignantschercheurs et doctorants qui se sont vu refuser un article pour ce motif.

3 À y voir de plus près et à force de lire les remarques des referees sur les pages refusées et donc retournées à l'auteur, les remarques " is not English ", "You should think in English », etc., recouvrent en fait deux grands types de reproches: d'une part, les membres des comités de lecture éprouvent quelque difficulté à trouver les résultats et, d'autre part et surtout, ils estiment que les auteurs francophones qui rédigent en anglais brûlent les étapes, escamotent des parties entières du raisonnement si bien que l'on a du mal à le suivre. Ce à quoi les auteurs francophones rétorquent, agacés, qu'ils ne vont pas «leur » refaire la science à chaque fois et « leur » démontrer une fois de plus ce que tout le monde sait déjà.

4 Nous sommes donc visiblement en présence d'un problème qui concerne les transferts de logique, et pour rejoindre mon propos premier, ce problème d'ordre logique ne peut pas être traité par traduction linéaire et encore moins par la traduction assistée. 


\section{L'article de recherche et la logicisation}

5 Rappelons que l'article de recherche est un genre textuel particulier (Swales 1990) au service de la communication entre pairs d'une même communauté scientifique. Le scientifique, c'est-à-dire le chercheur devenu homme-texte, se voit confronté au processus de stabilisation discursive d'une masse d'informations à ordonner.

6 S'il est en effet concevable que le contenu cognitif d'une recherche s'élabore dans un laboratoire, c'est-à-dire un lieu, des équipements et des inscripteurs (Latour \& Woolgar 1988 : 42), mais aussi une équipe de chercheurs travaillant selon une certaine méthode (consistant à vérifier/infirmer ou modifier expérimentalement une idée devenue hypothèse), il est, d'après nos informateurs, moins concevable d'en envisager le processus de construction de façon aussi linéaire et rationnelle. Différentes raisons sont en effet susceptibles de perturber cette démarche apparemment logique : le bruit, le doute qui parfois s'installe, les rivalités et soucis de carrière, les problèmes de financement et une démarche parfois empirique, sinueuse, voire instinctive.

7 Le contenu cognitif ne devient donc accessible que par le langage, c'est-à-dire «les réalisations et manifestations de la langue » (Culioli cité par Vignaux 1995 : 566) à travers une démarche logique fondée sur une argumentation cohérente et structurée. Cela revient à dire que la démarche logique et hypothético-déductive, fondée sur la justification expérimentale d'une redéfinition du réel, se retrouve dans l'article de recherche.

8 Or l'argumentation en général constitue le facteur essentiel de cette cohérence discursive qui suppose un enchaînement structuré d'arguments sans lequel il n'y aurait pas de stratégie globale. Car c'est bien de stratégie dont il s'agit puisque l'article de recherche vise à convaincre les membres de la communauté du bien-fondé scientifique de la thèse défendue pour que le pair y adhère.

9 L'article de recherche participe ainsi au processus de création de la connaissance par le biais d'agencements discursifs rendus logiques par une démarche de logicisation, ou logification (Martin 1996 : 16).

10 Cette logicisation du discours au sein de l'article de recherche appelle deux commentaires: le premier c'est que la logicisation consiste à tisser des liens logiques entre les objets et événements scientifiques de l'article, liens qui sont avérés par validation du comité de lecture (des pairs) ou directement par la politique éditoriale du journal (AIP Style Manual 1990) ; le deuxième c'est que la logicisation constitue également une démarche de persuasion (l'agonistique) puisque l'argumentation, en tant que suite justificative d'affirmations, s'insère dans un processus de communication visant à emporter l'adhésion du pair destinataire, voire à modifier ses convictions sur un sujet donné. Kocourek (1991: 60) parle alors de "cognitivité », c'est-à-dire le recours aux arguments rationnels.

11 Cette logicisation qui met en œuvre des arguments rationnels à effet persuasif, se manifeste ainsi dans ce qu'il est convenu d'appeler des stratégies argumentatives dont il m'a paru intéressant d'apprécier les différences entre auteurs francophones et anglophones, à partir de trente articles français et trente articles anglais consacrés à une discipline scientifique analytique peu étudiée jusqu'ici, à savoir la mécanique. 
Qu'il me soit permis de faire remarquer à cet égard que l'article de recherche en mécanique suit rarement et de façon atypique, le schéma canonique IMRD et j'ai donc analysé les rubriques apparentées à " discussion », " discussion ». Par ailleurs j'ai expliqué dans un article (Bachschmidt 1997) pourquoi $30+30$ articles étaient représentatifs à 99,9\% de la discipline considérée, si bien que les résultats de ma communication sont donc scientifiquement fondés.

\section{Différences entre le discours du francophone et de l'anglophone}

Avant de poursuivre, je voudrais préciser d'une part que ce qui va être dit sera fatalement caricatural en raison d'une profusion de rhétoriques individuelles, de comptages statistiques et de styles différents susceptibles d'induire des contradictions au niveau du détail des formes linguistiques, mais n'en demeure pas moins caractéristique de la modélisation de l'article de recherche en général; et que, d'autre part, ce qui va être dit expliquera aussi pourquoi les articles français comportent un nombre de mots inférieur de moitié au nombre de mots des articles anglais, à paragraphes sensiblement égaux.

En annexe, le lecteur trouvera sept extraits d'articles de recherche numérotés de 1 à 7 et dont les lignes numérotées de 5 en 5 lui permettront de trouver les références citées entre parenthèses.

\subsection{Rhétorique centrée sur les faits en français et rhétorique centrée sur l'argumentation en anglais}

a facticité du discours français se rencontre d'abord dans des énoncés où dominent des phrases peu reliées entre elles par des connecteurs logiques. Cette tendance à la juxtaposition syntaxique (asyndète) se manifeste donc dans un schéma appositif faisant tout de même intervenir quelques anaphores (ce qui, ceci, ce...).

Il s'ensuit une succession, voire une fragmentation en petits paragraphes plus ou moins autonomes coïncidant souvent avec une seule phrase et dont le caractère appositif est rendu par des mots de liaison du type "de plus» (1, ligne 27) introduisant une spécification (addition de détails supplémentaires). Chaque phrase-paragraphe est ainsi centrée sur un fait ou un événement scientifiques, comme si le chercheur francophone désirait avant tout communiquer un résultat ayant fait l'objet d'une expérimentation.

Il s'ensuit aussi un discours inscrit dans la chronologie (" Dans une première étape... dans un second temps ») et dans la temporalité liée aux marques de la personne: le passé composé avec « nous » (1, ligne 1$)$, le présent avec « on » (1, ligne 24) et le futur lié aux perspectives d'avenir comme c'est le cas dans un tiers des articles français pour un seul cas dans les articles en anglais (1, ligne 18).

discours de l'anglophone, par contre, se caractérise par l'extrême densité des liens interphrastiques car, de toute évidence, les auteurs anglophones veulent convaincre à l'aide d'une argumentation visant à éliminer tout ce qui pourrait être fortuit ou irrationnel.

19 Il s'ensuit une phraséologie complexe impliquant des propositions principales dans lesquelles sont enchâssées des propositions subordonnées ou plusieurs propositions 
subordonnées imbriquées. Ces propositions sont introduites par tout type de connecteur logique, de cause, de conséquence, de concession, d'hypothèse... (2, lignes 15 à $22 ; 3$, lignes 1 à 5).

\subsection{Discours de nature rhématique en français et discours de l'anglophone thématique}

(subordonnants) de cause : « because» (2, ligne 13), « as » (3, ligne 7), « since» (7, ligne 21 et 3, ligne 16) avec des formes causales en -ing; et de consécution : «so, thus, therefore »et «hence ». Si l'on suit Lapaire et Rotgé (1991), « so » par exemple sémantise le lien entre deux propositions en lui conférant un trait causal quasi naturel, «thus "possède souvent une valeur anaphorique de validation, de même que therefore comme l'a montré Vidalenc (1993: 396). 
29 Enfin, un large recours à l'assertion concédée marquée par la subordination restrictive avec essentiellement « although» qui renvoie à gauche (7, ligne 20). L'assertion concédée introduite par «although» sert à anticiper une opposition éventuelle de la part du destinataire.

\section{Au niveau interlocutif, quelle est la tâche du coénonciateur?}

Au niveau interlocutif des rapports entre l'énonciateur et le coénonciateur, le pair assure à la fois la tâche de destinataire de l'information contenue dans l'article de recherche et de coénonciateur (au sens où l'entend la théorie des opérations énonciatives - TOÉ - qui dit que tout énoncé est produit par un énonciateur à l'adresse d'un coénonciateur).

Celui-ci, en effet, est invité à admettre le contenu scientifique et donc cognitif de l'article de recherche, ainsi que les interprétations qui s'y rattachent.

Le coénonciateur francophone, de toute évidence, participe activement à la construction du discours car il est « contraint », disons « invité » à collaborer à l'élaboration du savoir en inférant une partie du travail logique implicité par l'énonciateur, les rapports de causalité en particulier.

33 À quoi se réduit la causalité dans les rubriques assimilées à "discussion » des articles français ? La relation causale dont j'ai déjà souligné le caractère peu récurrent est surtout marquée par les connecteurs « car, puisque et parce que ». «Car », seize occurrences dans notre corpus (6, ligne 3) rhématise l'énoncé: l'énonciateur assume la proposition justificative comme un motif possible de la véracité de la première proposition (la principale). «Puisque », dix occurrences (5, ligne 5) est un opérateur de thématicité (Adamczewski 1990 : 341) qui permet à l'énonciateur de se retrancher derrière une autre instance (la communauté des pairs) ou derrière le caractère évident du phénomène au regard des canons de la discipline. "Parce que», deux occurrences, introduit une justification rhématique incontestable et totalement prise en charge par l'énonciateur'1.

34 En d'autres termes, nous n'avons relevé que peu de connecteurs de cause dans nos 30 articles français, dont les deux tiers rhématisent l'énoncé, c'est-à-dire le fluidifient vers la droite. Dans ce contexte, on peut dire que l'énonciateur francophone ne veut pas toujours expliciter tous les rapports de causalité laissés à la discrétion inférante du coénonciateur.

La même remarque peut s'appliquer au phénomène dont nous avons parlé plus haut, à savoir la juxtaposition syntaxique qui oblige en quelque sorte le coénonciateur à inférer une partie du raisonnement.

36 Tel n'est pas le cas du coénonciateur anglophone qui n'est pas véritablement appelé à inférer quoi que ce soit et dont l'énonciateur anticipe systématiquement toutes les objections et critiques possibles en usant, comme nous l'avons vu, de toute la panoplie des liens argumentatifs pour qu'aucun élément informationnel ne soit laissé dans l'ombre. Tout est dit, même parfois les évidences scientifiques, selon nos informateurs.

37 Le coénonciateur anglophone n'est donc sollicité que pour covalider une information scientifique nouvelle dont il peut suivre la justification étape par étape. Comment pourrait-il d'ailleurs en être autrement puisque, si nous nous appuyons sur certains documents de référence en matière de "recommandations aux auteurs ", l'AIP Style Manual demande aux auteurs de se poser les questions suivantes: 
have I included all the information necessary to convey my message?

have I given proper emphasis to important ideas, and subordinated those of lesser importance?

is the development of the subject-matter logical and complete, free of gaps and discontinuities? homologuée Z 41-003 ( 1974) consacrée à la présentation des articles de périodiques, insiste sur la concision d'une information de consécution en ne mentionnant que l'évaluation comme mode de raisonnement :

- les résultats doivent être présentés de manière concise

- toute généralisation abusive est à éviter. Les modèles mathématiques ne doivent, en particulier, intervenir que comme conséquence d'un nombre suffisant de résultats.

Il s'ensuit un effacement de l'énonciateur anglophone au profit d'un raisonnement axé sur la facticité des objets ou événements scientifiques de l'article de recherche grâce à divers procédés linguistiques qui jouent en faveur des faits scientifiques :

- une tendance à la passivation permettant la focalisation sur le sujet grammatical, c'est-àdire les données scientifiques (7, lignes 6 et 20).

- L'emploi du mode performatif pour introduire des données qui, en position de sujet grammatical, acquièrent un certain degré d'animisation (Jacobi 1984 : 45) (7, lignes 11 - 13 et 17).

- Le recours au procédé de l'inversion pour créer un effet d'emphase (7, lignes 21 - 23).

- L'emploi de la forme « be+ing » à valeur modale et de nature interpellative (7, ligne 5 - 6).

- Le recours à des tournures impersonnelles (avec extraposition du sujet grammatical ayant pour effet d'occulter l'énonciateur).

- La présence de modaux, en particulier « will » et « can », qui permettent d'asserter l'existence d'une propriété ou la possibilité unilatérale.

L'énonciateur francophone, en revanche, demeure perceptible dans les marques de la temporalité comme nous l'avons vu, et surtout les marques de la modalité appréciative (Bouscaren \& Chuquet, 1987: 37) liée à des tournures impersonnelles du type «il est acceptable de supposer que... » (4, ligne $3-4)$.

\section{Conclusion}

41 L'assertivité du raisonnement des auteurs anglophones apparaît ainsi comme forte en raison d'une argumentation corroborative thématique rigoureuse et d'une cohésion discursive n'impliquant nullement le coénonciateur, pris à témoin d'une démonstration on ne peut plus explicite, par rapport à l'assertivité des auteurs francophones qui construisent leur argumentation à l'aide du coénonciateur.

Si l'article de recherche, autant français qu'anglais, a un objectif commun de persuasion, en tant que vecteur d'information scientifique, il n'en demeure pas un vecteur biaisé dans la mesure où s'y révèlent une appréciation différente de la réalité et une stratégie argumentative différente, en fonction de l'organisation des données de l'expérience qui correspondent à chaque langue et donc à chaque culture.

Il serait en effet intéressant de déterminer ces paramètres culturels (système éducatif, conscience collective du rôle international des États, avancées technologiques, etc.). De même serait-il intéressant de comparer plusieurs disciplines scientifiques à cet égard. 


\section{BIBLIOGRAPHIE}

Adamczewski, Henri. 1991. Le Français déchiffré, clé du langage et des langues. Paris : A. Colin. AIP Style Manual. 1993. New-York : Springer-Verlag.

Bachschmidt, Patrick. 1997. "Procédure de constitution d'un corpus attesté d'articles de recherche en vue d'une étude contrastive ». ASp 15-18, 133-138.

Culioli, Antoine. 1990. Pour une linguistique de l'énonciation. Gap : Ophrys.

Grize, Jean-Blaise. 1990. Logique et langage. Gap : Ophrys.

Latour, Bruno et Steve Woolgar. 1988. La vie de laboratoire ; la production des faits scientifiques.

Paris : La Découverte.

Loffler-Laurian, Anne-Marie. 1996. La traduction automatique. Paris : Presses Universitaires du Septentrion.

Martin, Jacky. 1996. «Les enjeux du discours scientifique : la stratégie de véridiction ». ASp 11-14, 13-31.

Norme française homologuée Z 41-003. 1974. Paris : AFNOR.

Kocourek, Rostislav. 1991. La langue française de la technique et de la science. Wiesbaden : Brandstetter.

Lapaire, Jean-Rémi et Wilfrid Rotgé. 1991. Linguistique et grammaire de l'anglais. Toulouse : Presses Universitaires du Mirail.

Swales, John. 1990. Genre Analysis. Cambridge : Cambridge University Press.

Vidalenc, Jean-Louis. 1993. «Un apport de l'analyse du discours à l'apprentissage de la rédaction d'articles scientifiques : l'étude des phénomènes de connexion ». ASp 1, 383-401.

Vignaux, Georges. 1995. Mélanges offerts à Antoine Culioli. Paris : Presses universitaires de France.

\section{ANNEXES}

\section{Références textuelles}


(1) Dans une première étape, afin de valider la méthode utilisée, nous avons envisagé des

[1. 1] vides de forme circulaire de façon à corréler les résultats obtenus numériquement

[1. 5] avec ceux obtenus analytiquement grâce à une méthode variationnelle utilisée par

HASHIN [AMI/90]. Dans un second temps nous avons envisagé le cas de vides

[1. 10] elliptiques, ce qui nous a permis d'évaluer l'influence du coefficient de forme à porosité

[1. 15] constante, pour différentes porosités, sur les coefficients C1111 et C2222 (Fig. 11 et 12)

caractérisant les rigidités dans les sens respectivement parallèle et perpendiculaire au

[1.20] grand axe de l'ellipse, ainsi que sur leurs rapports caractérisant l'anisotropie

[1. 25] mécanique.

On peut noter la similitude d'allure de la figure 13 avec celle de la figure 5 qui

traduisait la comparaison des conductivités thermiques sphères-ellipsoïdes en réseau

cubique simple. On note la même similitude entre la figure 14 et la figure 6 traduisant

l'évolution du rapport d'anisotropie thermique avec des ellipsoïdes en réseau cubique

simple.

Une modélisation numérique 3D permettra d'affiner la corrélation des

comportements mécanique et thermique dans des directions orthogonales l'une à

l'autre.

La figure 13 fait apparaître une limite maximale du rapport d'anisotropie voisine de 3. Ceci confirme les résultats obtenus expérimentalement sur matériaux réels [CAB-88a],

[CAB-88b]. [...]

On note que la rigidité dans le sens du grand axe de l'ellipse est supérieure à celle obtenue à même porosité avec un vide circulaire, alors que la rigidité dans le sens du petit axe de l'ellipse est inférieure. De plus cette dernière décroît rapidement lorsque le coefficient de forme augmente et ce, d'autant plus que la porosité est faible.

Cabrillac et al. 1992 « Influence de la porosité et de sa configuration sur le comportement mécanique et thermique des matériaux poreux anisotropes". Les Cahiers de rhéologie vol. $\mathrm{X} / 1,25-27$. 
(2) The high prestrain strain aging results plotted in Fig. 4 appear to be fitted reasonably

[1. 1] well by curves based on the Johnson-Mehl expression. The values of a for the data about

[1.5] the fitted curves were between 0.4 and $0.8 \%$ of strain aging, corresponding to $7.15 \mathrm{MN}$

$\mathrm{m}-2$, comparable to the within batch variation of the unaged material for the two

[1. 10] higher test temperatures, although the scatter is somewhathigher for the $80^{\circ} \mathrm{C}$

[1. 15] results. The rate parameter $m$ was found to lie between $0-28$ and $0-45$, with an estimated error of approximately +-0.1 . As with low prestrain strain aging, the rate

[1. 20] exponents show a temperature dependence, with the result at $80^{\circ} \mathrm{C}$, the lowest test temperature, being somewhat less than those at the two higher temperatures. The assumption of constant $\mathrm{P}_{\max }$ is probably more justified for these results than for the low prestrain results, because $\mathrm{P}_{\max }$ was obtained from the isothermal results (at $120^{\circ} \mathrm{C}$ ). The calculations provided activation energy values greater than those expected for interstitial solute diffusion in ferrite. The highest minimum and lowest maximum values from Table 5 give a value for $Q$ between 109.4 and $113.0 \mathrm{~kJ}$ mol-l. Therefore, the results do not appear to support solute segregation to dislocations as the rate limiting process, although, again, the method of determination of $Q$ is strictly only reliable when the same rate exponent is found at each test temperature.

Bramley et al. 1990. "Static Strain Aging in high Carbon Steel Wire". Materials Science Technology vol. VI/4, 336 .

\begin{tabular}{|c|c|}
\hline (3) [l, 1] & $\begin{array}{l}\text { These results suggest that at large values of torsional stiffness, only the } \\
\text { stable center at the switching fine } \Psi=0 \text { remains, and thus the system is } \\
\text { always stable (provided that } \mu<\mu_{c 1} \text { ). However, the particular value of } \Omega \\
\text { at which the unstable equilibrium point disappears also depends on other } \\
\text { variables, particularly on } \mu \text {. } \\
\text { The effects of the length-to-width ratio of the mass are similar to those of } \\
\Omega \text {, but with one difference. As y is increased, the stable and unstable pairs } \\
\text { move simultaneously toward the origin instead of away from it, leaving a } \\
\text { smaller stable region about the origin. }\end{array}$ \\
\hline$[1,15]$ & $\begin{array}{l}\text { Contrary to the effects of } \Omega \text { and } y \text {, increasing values of the static normal } \\
\text { force } F_{s,} \text { repel the stable and unstable points away from each other. The } \\
\text { saddle point moves toward the origin (without ever actually reaching it), } \\
\text { and the stable equilibrium point moves away from the origin. The end result } \\
\text { corresponds to a decrease in the domain of attraction of the stable point } \\
\text { at the switching fine, l.e., the origin. From a design point of view, this } \\
\text { Il.15jis not desirable since in most cases a large domain of attraction near } \\
\text { the origin is preferred for stable behavior. Therefore, the smallest } \\
\text { possible value of } F_{s r} \text { would be desirable as permitted by other system } \\
\text { requirements for optimization. } \\
\text { Akay et al. 1994. "Effects of System Dynamics on Friction-induced } \\
\text { Oscillations". Joumal of Sound and Vibration, } 606 \text {. }\end{array}$ \\
\hline (4) [l. 1$]$ & 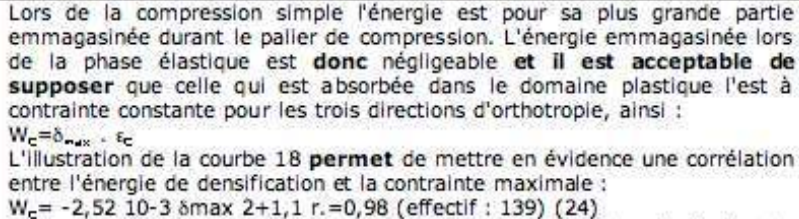 \\
\hline [l, 15] & $\begin{array}{l}\text { Cette représentation permet de regrouper les trois directions d'orthotrople. } \\
\text { La relation (24) révèle que pour une contrainte maximale inférieure ou } \\
\text { égale à } 15 \mathrm{MPa} \text { on retrouve les résultats obtenus pour les directions } \\
\text { transverses, le premier terme de l'équation devenant négligeable et } \\
\text { réduisant alors le comportement a une relation d'ordre } 1 \text {. Au-delà de } 15 \\
\text { MPa, les résultats de la direction longitudinale s'inscrivent dans la continuité } \\
\text { des précédents, l'énergie de densification semble alors tendre vers un } \\
\text { palier lorsque smax dépasse } 60 \mathrm{MPa} \text {....] } \\
\text { Morlier et al. 1993. \& Plasticité du bois en compression simple \#. Matériaux } \\
\text { et Techniques vol. } 44 / 2,12-13 \text {. }\end{array}$ \\
\hline
\end{tabular}


(5) On constate néanmoins que les trois courbes sont sensiblement confondues sur la partie

[1. 1] du cycle correspondant à la phase de compression des gaz, lorsque les oscillations

[1.5] parasites ont disparu par suite de l'amortissement. Le dispositif d'équilibrage y est donc bien efficace, puisque la chute de vitesse due à la compression des gaz (voir trace A) a disparu.

Caignaert. 1994. «Limitation des vibrations des machines alternatives par réduction de l'irrégularité cyclique de rotation ». Mécanique industrielle et Matériaux vol. 44/2, 292.

(6) Nous avons aussi calculé la limite géométrique du cisaillement selon une formule

[1. 1] donnée par Skelton[6] (lignes pointillées sur la Figure 2). Cette limite ne peut être asymptote pour nos courbes car les deux modèles sont entièrement différents.

[1.5] Cependant les résultats de Skelton pourraient être considérés comme une bonne approximation. Au-delà de cet angle de cisaillement la compression latérale entre fils pourrait entraîner des plis dans le plan du cisaillement. Ce phénomène est assez fréquemment observé lors des essais de cisaillement.

Drean et al. 1995. « Modélisation du comportement mécanique d'un tissu toilé ». Actes du XII Congrès français de mécanique vol. 2, 48.

(7) Saddoughi and Joubert (1991) reported peak values of a1 of about 0.14 in a laterally

[1. 1] diverging boundary layer, as compared with 0.14-0.15 in a simple 2-D boundary layer with low free-stream turbulence. Results for the present study, shown in Fig.11, are somewhat lower, except at $\mathrm{z} / \mathrm{s}=1.238$ where the boundary layer is only rnildly

[1.10] converging and is relatively unaffected by free-stream turbulence. For the data points

[1. 15] closest to the wall at other $\mathrm{z} / \mathrm{s}$, al is roughly $0.10-0.11$, generally consistent with

Saddoughi's results close to the wall. However farther away from the wall falls,

[1.20] gradually at first and then more rapidly for $y / 995>0.3-0.4$. This is qualitatively in

[1. 25] accordance with the results of Hancock and Bradshaw (1989), which indicate a drop in

[1.30] a1 in the outer half of the layer due to the effects free-stream turbulence (which contributes to $q^{\prime 2}$ but not to $\left.-u^{\prime} v^{\prime}\right)$. An additional factor in evaluating the present data is the uncertainty in determining 995 beneath the vortex (see Section 2.2) - therefore 995 may be overestimated or, inappropriately large measure of boundary layer thickness.

Figure 12 shows the $(1,2)$ and the $(2,3)$ components of the eddy viscosity, obtained at $\mathrm{x} / \mathrm{s}=6.24$. In fact all the components of eddy viscosity were calculated, although they are not all presented. Since for the 1,1 component, and possibly others, $\mathrm{x}$-derivatives were important, all results were interpolated from planes at $\mathrm{x} / \mathrm{s}=5.81$ and $\mathrm{x}=6.67$. Also shown in the Figure is the outer layer model Cebeci and Smith (see e.g., Cebeci and Bradshaw 1977). In accordance with the results of previous workers, the 1, 2 component is high in the laterally diverging portion of the boundary layer and low in the laterally converging portion. The 2,3 component is shown only at $\mathrm{z} / \mathrm{s}=-0.857$, and (in the outer part of the layer) being so small at other locations that the eddy viscosity is ill-posed. It is in agreement with the 1,2 component close to the surface, but Iower further out. The 1,2 and 2,3 components of eddy viscosity would be equal if the boundary layer were two-dimensional or collaterally divergent.

Bradshaw et al. 1993. "Strong Vortex Boundary Layer". Experiments in Fluids 14, 329. 


\section{NOTES}

1. Par opposition, nous avons dénombré 87 because, 75 since et quelques for causatifs.

\section{RÉSUMÉS}

Dans le présent article, il s'agit de montrer comment le chercheur francophone ou anglophone, devenu homme-texte (énonciateur), construit son argumentation dans l'article de recherche pour justifier sa démarche expérimentale de façon logique auprès de ses pairs (coénonciateur). Il s'agit également de voir comment le discours du francophone se fonde sur une rhétorique rhématique centrée sur les faits et un raisonnement déductif alors que l'anglophone recourt à une phraséologie complexe de nature thématique et inductive. Il s'agit enfin d'analyser la manière dont l'énonciateur et le coénonciateur cohabitent dans un discours à forte valeur stratégique

This article attempts to show how a French-speaking and an English-speaking researcher assumed to be the utterer of a text - build up their arguments in the research article so as to justify their experimental approach to their peers - as the co-utterer - in a logical way. It also studies how the French-speaking researcher's discourse unrolls on a rhematic rhetoric focused on facts and based on deductive reasoning, as compared to that of the English-speaking researcher who uses a complex topical and inductive phraseology. The article also analyzes how the utterer and co-utterer interact within a highly strategic discourse.

\section{INDEX}

Mots-clés : assertivité, coénonciateur, discours, raisonnement (déductif et inductif), rhématique, thématique

Keywords : assertivity, co-utterer, discourse, reasoning (deductive and inductive), rhematic, topical

\section{AUTEUR}

\section{PATRICK BACHSCHMIDT}

Patrick Bachschmidt est titulaire d'un doctorat d'anglais de spécialité de l'Université Montpellier 3 consacré au discours scientifique. Il est responsable du département des langues et cultures étrangères de l'École nationale supérieure des industries textiles de Mulhouse, Université de Haute-Alsace (ENSITM). patrick.bachschmidt@uha.fr 